Radiologe $2020 \cdot 60: 1-5$

https://doi.org/10.1007/s00117-019-00628-7

(c) Springer Medizin Verlag GmbH, ein Teil von Springer Nature 2019

\section{J. Herold' ' S. Delorme ${ }^{2}$}

' Universitätsklinik für Radiologie und Nuklearmedizin, Medizinische Universität Wien, Wien, Österreich

${ }^{2}$ Abteilung Radiologie (E010), Deutsches Krebsforschungszentrum (DKFZ), Heidelberg, Deutschland

\title{
Brave new world
}

Liebe Leserinnen und Leser,

ein Gespenst geht um in der Radiologie - das Gespenst der künstlichen Intelligenz (KI). Unser Fach steht vor einer Zeitenwende, an der alles anders werden kann. Zeitenwenden gab es zuvor, angefangen mit der Entdeckung der Röntgenstrahlen und der Geburt der Radiologie, über die Einführung von Kontrastmitteln bis hin zur Etablierung der Schnittbildverfahren und ihrer ungeahnten Möglichkeiten, aber noch nie zuvor war die Entwicklung von so viel Unsicherheit begleitet, teils berechtigt, teils nicht. Fest steht, dass niemand am Thema der künstlichen Intelligenz und des maschinellen Lernens (ML) vorbeikommt, zu präsent ist es in den Medien, in Betrachtungen zur digitalen Revolution 4.0 in der Industrie und in der radiologischen Fachliteratur ohnehin. Mehr noch: Will man nicht, dass der Zug nach eigenen Gesetzmäßigkeiten und allein gemäß dem freien Spiel der Kräfte rollt, ist es höchste Zeit, aufzuspringen und die Herausforderungen die wissenschaftlichen wie die regulatorischen - anzunehmen. Und ohne dass es aufgefallen wäre, hat er hat bereits mächtig Fahrt aufgenommen, gewissermaßen auf den Schienen von „Big Data“. Während in der Radiologie noch die Chance besteht, diesem datenverschlingenden Monster Form und Regeln zu geben, z.B. in Form von Datenrepositorien, ist es im Alltag der gesellschaftlichen Kontrolle bereits weitgehend entglitten, indem wir alle ihm bereitwillig unsere Daten überantworten, in Webshops und Suchmaschinen, durch Positionsdaten unserer Smartphones und nicht zuletzt in sozialen Netzwerken. Wie rieb man sich ungläubig die Augen, als durchsickerte, wie vor drei Jahren die unselige
Allianz zwischen Facebook und Cambridge Analytica maßgeschneiderten Wahlkampfkampagnen in Europa und den USA zugearbeitet hatte!

Man kommt also an diesem „artificial hype " kaum vorbei. Zeit also, angesichts der breiten Palette unterschiedlichster Zukunftsszenarien - vom kompletten Ersatz „menschlicher Radiologen“ durch diverse Algorithmen bis hin zur Erkenntnis der Notwendigkeit der künstlichen Intelligenz für die Weiterentwicklung des Faches selbst - ein Themenheft des Radiologen zu gestalten. In diesem Sinne haben wir versucht, Ihnen einen möglichst breit gestreuten Überblick über die mit künstlicher Intelligenz und maschinellem Lernen verbundenen Begriffen, deren technische und mathematische Grundlagen bis hin zu bereits im Entstehen begriffenen Anwendungsgebieten in möglichst verständlicher Form zu geben. Als Heftherausgeber haben wir selbst in einem intensiven Diskurs mit den jeweiligen Autoren versucht, alle Inhalte zu verstehen, möglichst verständliche Erklärungen und Formulierungen zu finden und mitzugestalten.

Dabei ist künstliche Intelligenz aus unserer, an den rasenden Fortschritt adaptierten Sicht eigentlich ein uralter Begriff. Der Begriff „artificial intelligence“ (AI) wurde bereits in den 50er Jahren verwendet, eigentlich als Folge der Konzepte für den Bau der allerersten Computer. Bereits in den 1980er Jahren des letzten Jahrhunderts wurde in Wien eine Professur für Kybernetik und künstliche Intelligenz geschaffen; seit 1984 existiert eine österreichische Gesellschaft für „artificial intelligence“. In einer nächsten Welle gab es große Anstrengungen, mit computer-assisted detection/diagnosis(CAD-)Softwareprogrammen Radiologen in ihrer Arbeit beispielsweise zur 
Detektion von fokalen Veränderungen der Mamma bzw. Lungenherden zu unterstützen. Der Durchbruch der künstlichen Intelligenz bzw. des maschinellen Lernens in den letzten Jahren ist neben algorithmischen Weiterentwicklungen jedenfalls auf die modernste Computerhardware mit "graphics processing units" (GPU) zurückzuführen. Die Rechenleistung der modernen Computer hat dazu geführt, dass riesige Datenkollektive durchforstet, verborgene Zusammenhänge zwischen Strukturen und Parametern entdeckt, Korrelationen mit Bilddaten und genomischen Daten durchgeführt und Krankheitsverläufe für Patienten vorausgesagt werden können.

In der Tat beflügeln diese enormen Rechenleistungen bei den digitalen Riesen und natürlich auch bei Sanierern im Gesundheitswesen und diversen Krankenhausadministratoren Phantasien, dass Radiologen über kurz oder lang entbehrlich werden könnten, ersetzt durch Algorithmen, die man mit den Millionen Bildpunkten aus dem Datensatz des menschlichen Körpers füttert, um dann eine objektivierbare, reproduzierbare, von menschlichen Faktoren wie beispielsweise Müdigkeit, schlechter Laune, Wunschdenken oder Euphorie unabhängige Diagnose zu erhalten. Sie mögen gerne weiter träumen. Bislang zumindest zeigen diverse Studien zwar das große Potenzial maschinellen Lernens, aber auch die Schwierigkeiten und Limitationen derartiger Verfahren. So ist es zwar Algorithmen möglich, bestimmte Bildinhalte, v. a. auf zweidimensionalen Datensätzen, zu identifizieren. Andererseits ist es derzeit (noch) nicht möglich, die Komplexität eines Lungenröntgens oder eines Abdomen-CT so zu erfassen, dass dabei eine klinisch sinnvolle Diagnose herauskommt. Es ist unsere Überzeugung, dass wir als Diagnostiker nackt dastehen, solange wir allein auf die Bilder zurückgeworfen sind, bar aller Informationen über Anamnese und klinische Befunde. Die Software, welche die urärztliche Leistung des integrativen und menschlichen Denkens stemmt, muss erst noch geschrieben werden. Dessen ungeachtet bleibt wohl, so lange die KI allein wegen eines farbigen Kärtchens neben dem zu erkennenden
Objekt statt einer Banane einen Toaster sieht [1], eine gewisse fachliche Aufsicht vonnöten, sofern man nicht bereit ist, vergleichbare Patzer in der Medizin als Kollateralschaden hinzunehmen.

Hinter den Sammelbegriffen maschinelles Lernen und „artificial intelligence“ verbergen sich zahlreiche Kategorien und Subkategorien. So beschäftigt sich die diagnostische künstliche Intelligenz mit Verbesserung der Diagnostik und der Treffsicherheit bei eng umrissenen Fragestellungen - also dem radiologischen Kerngeschäft, Detektion und Differenzialdiagnose. Prädiktive künstliche Intelligenz ist eine neue Entwicklung, die sich mit der Identifizierung neuer Biomarkern aus großen Datensätzen beschäftigt und die als Voraussetzung für die Weiterentwicklung der personalisierten Medizin gilt. Biomarker können aus Labordaten, klinischen Parametern, pathologischen Befunden, genetischen und molekularen Daten sowie Bilddaten stammen. Wenn nun von Bilddaten die Rede ist, so geht dieser Begriff über das hinaus, was unser gegenständliches Denken in Bildern erkennt, wie Organen oder Herdbefunden, und umfasst Merkmale, die wir nicht oder nur vage erahnen und schon gar nicht zwischen verschiedenen Patienten vergleichen können, wie Textur, Homogenität oder quantitative Formmerkmale, die aber mit mathematischen Methoden sehr wohl objektivierbar sind. Strenggenommen werden hierfür nicht einmal Bilder benötigt - diese werden nur von uns Menschen benötigt -, sondern es reichen unter Umständen die Rohdaten. Wir erleben gerade, wie die Texturanalyse eine Renaissance erfährt, mit der in den 1980er und 90er Jahren an Ultraschallaber auch an CT-Daten wissenschaftlich gearbeitet wurde, die aber mangels rechnerischer Ressourcen für eine klinische Implementierung eingeschlafen ist. Diese enorm großen, heterogenen (klinischen, laborchemischen, genetischen, radiologischen) Datensätze werden auf Zusammenhänge hin analysiert, die für das menschliche Auge und Gehirn nicht fassbar sind, um Subpopulationen von Patienten mit ähnlichen Merkmalen und ähnlichen klinischen Verläufen nach Therapie $\mathrm{zu}$ identifizieren und $\mathrm{zu}$ prognostizieren. In der Genetik arbeitet man hiermit schon lange, daher die neuen Buzz-Words „Radiogenomics“ oder "Radiomics“.

Operationale künstliche Intelligenz bezieht sich auf die Nutzung von Daten aus Krankenhausinformations- und Radiologieinformationssystemen zum besseren Verständnis und zur Verbesserung von Workflow, Reduktion von Wartezeiten und besserer Ausnutzung des bestehenden Medizintechnikparks. „Conti-

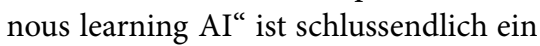
Begriff, der das ständige Füttern der Algorithmen durch neue Daten, wie eben im Krankenhaus im Allgemeinen oder einer radiologischen Klinik im Besonderen, in Echtzeit beschreibt.

Eine weitere Anwendung der KI hat noch keinen Namen, nämlich die Unterstützung der Bilderzeugung aus schwachen Rohdaten. Sowohl für die MRT, mit Blick auf die Geschwindigkeit, als auch für die CT in Hinsicht auf den Strahlenschutz verlockend: aus Rohdaten mit hohem Rauschanteil unter Zuhilfenahme von KI-Algorithmen aussagekräftige Bilder zu generieren.

Es wird nichts helfen, die KI als Teufelszeug zu verdammen und im Schmollwinkel zu verharren. Wenn nicht die Radiologie- als naturgemäß digitalstes aller Fächer - die Entwicklung aktiv gestalten hilft, wird die KI im freien Spiel der Kräfte ihren Raum greifen, mit womöglich zweifelhaftem Resultat. Glücklicherweise zeigt die Radiologie eine erstaunliche Konsequenz, die KI zu integrieren. So waren in den letzten beiden Jahren auf allen großen Kongressen unseres Faches KI- und ML-Themen massiv präsent, die Sitzungen, die sich mit diesem Thema beschäftigen, überfüllt und die Industrieausstellungen geprägt von einer intensiven Beschäftigung mit diesem Thema inklusive der Erkenntnis, dass zahllose Start-up-Unternehmen entstehen. Die Radiologie hat also die Herausforderung angenommen, eher noch als andere medizinische Fächer, denen die digitale Revolution wohl erst passieren wird.

Was sind nun die größten Herausforderungen und Trends? Wenn Radiologen der KI eine Rolle in ihrem Alltag zugestehen sollen, müssen sie in Grundzügen verstehen lernen, was sie tut, ohne gleich 
Hier steht eine Anzeige.

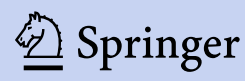


zu Informatikern zu mutieren. Niemand wird sich auch nur ansatzweise auf ein System verlassen, das eine völlige Black Box ist. Dabei dürfen wir uns keinen Illusionen hingeben: Zwar werden wir verstehen können - anfangsweise vielleicht auch mit Hilfe dieses Heftes -, was ein KISystem tut, aber nicht einmal der Schöpfer der Software wird sagen können, warum genau das System so oder so entscheidet. Es liegt in der Natur des Lernprozesses, zumindest beim unüberwachten maschinellen Lernen, dass sich das System seine eigenen Wege zum Ziel sucht und wir als einzige Kontrolle nur prüfen können, ob es auch dahin findet. Das Problem der „Verstehbarkeit“ ist indes in der „Szene" inzwischen angekommen. Inwieweit Anwender oder Wissenschaftler künftig die "gedanklichen“ Wege des Systems werden nachvollziehen können, ist eine spannende Frage. Es würde die Akzep$\tan z$ in jedem Fall stärken. Aus rezenten Studien wissen wir, dass bei Radiomics-Algorithmen bis zu 120 sog. Features identifiziert werden können, welche einen Lungenherd und dessen peritumorale Umgebung charakterisieren können. Allerdings können wir zum gegenwärtigen Zeitpunkt nur einen Bruchteil dieser 120 Parameter und ihrer Zusammenhänge, die von Algorithmen genutzt werden, morphologisch und funktionell zuordnen. Nicht dass wir dümmer wären, wir wenden nur weniger Kriterien an, die dafür umso differenzierter sind. Besinnen wir uns aber darauf, wo wir beim Umgang mit KI eigentlich gefordert sind: Wissen, was sie grundsätzlich tut, und warum und wie sie irren kann. Wir benötigen ein neues Risikobewusstsein und -management, um systematische Irrtümer und möglicherweise katastrophale Folgen für Patienten zu vermeiden. Je breiter der Einsatz, desto größer ist die Gefahr blinden Vertrauens.

An den Umgang mit großen Datenmengen und potenten Algorithmen sind hohe ethische Ansprüche zu stellen. Die Daten, die Patienten uns überlassen, welcher Art auch immer, sollen ausschließlich der Weiterentwicklung der Medizin im Interesse von Patienten zugutekommen, unabhängig davon, ob sie in der Hand der Wissenschaft oder der Industrie sind. Dies erfordert auch die Be- achtung strengster Regeln hinsichtlich Datensicherheit, Privatsphäre und Patientenkonsensus. In diesem Zusammenhang wird zunehmend der Begriff einer humanistischen künstlichen Intelligenz verwendet, als europäischer Gegensatz zu einem eher geschäftsorientierten transatlantischen Modell. Dass dies notwendig ist, zeigt ein Blick auf rezente Statistiken, welche die Entwicklung des maschinellen Lernens und der künstlichen Intelligenz nahezu ausschließlich im Volumen der umgesetzten Dollars misst.

Wer trägt die Verantwortung für KIunterstützte oder KI-basierte radiologische Befunde? Das derzeitig gültige Rechtsverständnis geht davon aus, dass Maschinen eine solche Verantwortung nicht übernehmen können - vorerst also wird die Verantwortung bei der anwendenden Person verbleiben. $\mathrm{Zu}$ einen impliziert dies die Pflicht zur kritischen Wertung automatisch erzeugter Befunde, zum anderen aber auch regulatorische Anforderungen, denn wer KI einsetzt, muss sich darauf verlassen können, dass sie hohen Qualitätsanforderungen genügt: Eine Herkulesarbeit für zertifizierende Organisationen wie FDA und EMA, aus den tausenden in Forschungsinstituten entwickelten Algorithmen, die für ein Medizinprodukt tauglichen herauszufinden, die wiederum -als wesentliche Voraussetzung eines breiten Einsatzes von ML und KI im klinisch radiologischen Alltag - von den großen Medizintechnikfirmen in ihren Produkten bzw. ihrer Softwareunterstützung integriert werden können.

Solange die Möglichkeit besteht, maschinell erzeugten Befunden medizinischen Sach- und gesunden Menschenverstand entgegenzustellen, lassen sich Fehler abwenden: Entgangene Rundherde, falsch-positive Befunde, fehlerhaftes TNM-Staging, verfehlte Prognosen - alles kein Problem, solange ein fachlich qualifiziertes menschliches Wesen kritisch darüber schaut. Aber was, wenn hierzu die Möglichkeit fehlt? Die Rede ist vom Einsatz der KI in der Bilderzeugung, nicht der Bildinterpretation. Die Entwicklung von Methoden, um aufgrund von Rohdaten schlechter Qualität immer noch aussagekräftige Bilder zu erzeugen, ist verlockend, denn so können in der CT Strahlendosis und in der MRT womöglich Messzeit eingespart werden. Nur: Hier ist die Rettungsleine endgültig gekappt. Aufgrund der geringen Qualität der Rohdaten gibt es keine Möglichkeit, die Richtigkeit der berechneten Bilder oder auch der automatisch erzeugten Befunde zu prüfen. Solche Verfahren berühren nicht nur empfindlich das Nutzen-Risiko-Verhältnis, sondern können durch falsch-positive, negative oder einfach falsche Bilddetails unmittelbaren Schaden verursachen. So begrüßenswert diese Entwicklungen sind, bedürfen sie doch dringend nicht nur der kritischen wissenschaftlichen Diskussion, sondern einer scharfen Risikoanalyse. Auch stellen sie ein bislang so nicht bekanntes regulatorisches Problem dar, das in Angriff genommen werden sollte, bevor die Entwicklung und Implementierung dem zuvorkommt.

Die Sirenen singen uns, dass eine KIunterstützter Workflow mit von KI-Algorithmen vorselektionierten Differenzialdiagnosen und Entscheidungshilfen uns den Alltag so erleichtern werden, dass uns mehr Zeit für andere Aufgaben wie beispielsweise die patientenzentrierte Radiologie und die Kommunikation bleibt. Lassen wir uns nicht täuschen: Es wäre das erste Mal, dass uns jemals eine neue und komplexe Technik Arbeit erspart hätte. Freiräume, sollten sie sich je auftun, werden umgehend gefüllt, und dass wir unsere Arbeit mittels der KI absichern, wird nebenbei selbstverständlich gefordert werden. Denken wir nur an die Digitalisierung, der daraus resultierenden, sich immer schneller drehenden Informationsspirale, das E-mailStakkato, den Zeitaufwand, der aus der Beschäftigung mit elektronischen Krankengeschichten entsteht $(, \ldots$ why doctors hate their computers ...") sowie den aus der Digitalisierung entstandenen, immer komplexer werdenden Bildinformationen. Kann es sein, dass uns Maschine Learning und Artifical Intelligence in eine neue Dimension der Komplexität führen, deren Analyse und Beurteilung unsere Kapazitäten weiter überfordert?

Was immer die Herausforderungen sind, bleibt uns Radiologen keine Wahl, als uns intensiv mit dem Thema zu beschäftigen. Der beste Zugang zu einem 
neuen Werkzeug ist es, dieses zu nutzen und nicht jemand anderem zu überlassen. Womöglich wirken wir, wie Schwarzseher warnen, als nützliche Idioten an unserem eigenen Untergang mit, aber, frei gesprochen, gibt es keine Alternative zum aktiven Gestalten - auch wenn sich die Arbeitswirklichkeit der kommenden Generation von unserer unterscheiden wird. Und wer glaubt, durch Serverfarmen Radiologen ersetzen zu können, ohne anatomisches, morphologisches, biochemisches, molekulares, pathologisches und radiologisches Wissen, und ohne dem Klinker einen mitdenkenden Partner an die Seite zu stellen, dem seien zwei Dinge entgegengehalten: einmal die gemeinsame Sehnsucht von Ärzten und Patienten nach einer menschlichen Medizin, und zum anderen ein Satz von Will Thurlback, einem großen amerikanischen Pathologen: „Technology should not be used for its own sake: a fool with a tool is still a fool ....".

In diesem Sinne wünschen wir Ihnen eine interessante Lektüre in einem der spannendsten Themenhefte, die uns in Bezug auf neue Methoden in den letzten Jahren gelungen ist. Wir bedanken uns insbesondere bei allen Autoren, welche in einem sehr intensiven Interaktionsprozess verständliche Artikel zu zahlreichen Teilaspekten geschrieben haben, von denen wir in den letzten Wochen bereits viel lernen konnten, und nicht zuletzt bei unserem Verlag, der angesichts dieses aufwändigen Prozesses sehr viel Geduld mit uns hatte!

Ihre

Prof. Dr. Christian J. Herold

Prof. Dr. Stefan Delorme

\section{Korrespondenzadresse}

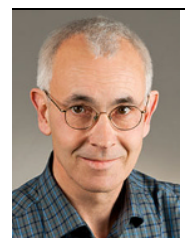

Prof. Dr. S. Delorme

Abteilung Radiologie

(E010), Deutsches

Krebsforschungszentrum

(DKFZ)

Im Neuenheimer Feld 280,

69120 Heidelberg,

Deutschland

s.delorme@

dkfz-heidelberg.de
Interessenkonflikt. C.J. Herold und S. Delorme geben an, dass kein Interessenkonflikt besteht.

\section{Literatur}

1. https://www.zeit.de/2019/47/kuenstlicheintelligenz-neuronale-netze-muster-erkennungforschung $\frac{\text { Autoren }}{\text { WERKSTATT }}$

GRATIS

Ein Service für Autoren von Springer Medizin

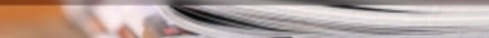

\section{Fortbildungen für Autoren und Gutachter}

Die ersten Veröffentlichungen sind für jeden Mediziner ein wichtiger Schritt in seiner Karriere als Wissenschaftler. Wissenschaftliche Artikel sind entscheidend dafür, dass die eigene Arbeit in der Community wahrgenommen wird. Es geht darum, die eigenen Ideen national und international auszutauschen und sicherzustellen, dass die Ergebnisse Wirkung erzielen. Die Online-Kurse der Autorenwerkstatt helfen, sich leicht einen Überblick über das Schreiben, Einreichen, Begutachten und Veröffentlichen eines Manuskripts zu verschaffen.

5 Online-Kurse zu den wichtigsten Standards des wissenschaftlichen Publizierens:

- Wie verfasse ich ein Manuskript?

- Writing in English für deutschsprachige Autoren

- Wie funktionieren Publikation und Begutachtung?

- Anleitung zur Open-AccessVeröffentlichung

- Leitfaden zur Peer-ReviewBegutachtung

Für alle, die auf SpringerMedizin.de registriert sind!

Jetzt gratis fortbilden unter www.springermedizin.de/ autorenwerkstatt/ 